\title{
Forum
}

\section{Misreading the conservation landscape}

\begin{abstract}
The social sciences are often seen as being in opposition to conservation and the practice of conservationists. Yet social scientists have made important contributions to conservation and could make even more contributions if they are willing to use their perceptive, insightful tools as a means of both improving the practice of conservation and sharpening social science's critique of conservation ideas and practices. I provide two lists: first, a list of the ways in which I think social science work has already improved conservation practice and, second, a set of generalizations made by some social scientists about the practice of conservation that are incorrect or incomplete. I argue that a more careful application of social science tools and approaches could begin an active and informed exploration of the diversity of values, histories, institutions, politics and approaches in conservation. This would facilitate the sharpening of social science's critique of conservation ideas and practices and, through these, improve the practice of conservation.
\end{abstract}

Keywords Collaboration, conservation, social sciences

\section{Introduction}

Tn a seminal book social scientists Fairhead \& Leach (1996) 1 demonstrated how forestry officials and conservationists misread the effects of local peoples on the extent of forest cover in the Republic of Guinea. Using painstaking fieldwork incorporating the biological, social, and historical sciences they concluded that instead of being forest destroyers, these people were responsible for increases in forest cover: their role had been completely misjudged. Fairhead \& Leach's approach is exemplary in using careful scholarship and informed interpretation to understand local practices, beliefs and behaviours: an approach to inquiry that appears to have been forgotten by some of the social science community writing about the practice of conservation.

I write here as a practising conservationist trained as a biologist. Over the last few decades I have learned a great deal about my own discipline and practice through reading the works of, and collaborating with, social scientists. Building on my experience I here make the case for a more strategic, systematic, open-handed collaboration between

Kent H. Redford Wildlife Conservation Society, 2300 Southern Boulevard, Bronx, NY 10460, USA. E-mail kredford@wcs.org

Received 24 September 2010. Revision requested 8 December 2010. Accepted 4 January 2011. conservation practitioners and social scientists (focusing on anthropology, political science and geography). The objective of such a collaboration would be to create a resilient practice that conserves the world's biodiversity while respecting and empowering people. Such a practice will benefit not only from the direct application of improved practices but, indirectly, from the careful study of practice, practitioners and the culture of both. I suggest two things: firstly, that some of the methods whose use has led to so many significant advances in social science are not being used to best advantage in studying conservation and, secondly, that careful empirical work on conservation practice by social scientists can better inform the practice of conservation.

We are on a common journey shaped by the growing power of human influence on both nature and the people whose lives are most directly intertwined with the natural world. Long-standing differences that typify those of us who came to this field decades ago are not necessarily shared by a younger generation, trained to think across disciplinary boundaries and with evolving sets of values. Here I hope to help lay the ground work for a stronger collaboration between disciplines, between discipline and practice, and in the training of the next generation of those interested in the fate of the natural world (Mascia et al., 2003).

As a means of advancing this agenda I provide two lists. First is a list of the ways in which I think social science work has already improved conservation practice. Second is a set of generalizations made by some social scientists about the practice of conservation that are incorrect or incomplete. Sometimes these are made explicit and sometimes they remain implicit. In both cases they deserve considerably greater examination and, if reconsidered, may further strengthen the contribution that social science could make to conservation practice. The first list shows the power of the partnership I advocate and the second points to what I think are the most important things to address to better inform collaborative efforts between social scientists and conservation practitioners. These lists are not meant to be exhaustive but illustrative. I am sure that others may make lists different from mine.

Not all social sciences are the same and neither are all social scientists. The same is true for conservationists and I am aware that what I call 'conservation' is a diverse family. Equally true is the gradual dissolving of the distinction between us-the conservationists-and them-the social scientists. Yet despite this, there remains an apparent anticonservation orthodoxy in some of the social science literature 
that, in my opinion, is inhibiting improved conservation practice, greater collaboration, greater incorporation of key social science values and methods into conservation practice, and improved interdisciplinary training of young social scientists and conservation practitioners. I hope to both applaud what I think are some of the contributions the social sciences have made to conservation and to highlight some of the generalizations that I think are impeding further constructive engagement. I have not provided extensive citations as I wish to focus on a general reading of the literature.

\section{Ways in which the social sciences have improved conservation practice}

Showing that conservation is more than biology The contemporary practice of conservation was born out of conservation biology, where its roots have remained. Most practising and academic conservationists were trained in various disciplines of the natural sciences. Conservation organizations have promoted science-driven approaches that have largely ignored the social sciences. Perhaps it was an act of hubris on our part to assume that such training equipped us to practice conservation but we were the only ones stepping forth, and so we did. As a result conservation practice has been based on biocentric values and assumptions, privileging natural science views of both problems and solutions. But we have learned that conservation practice is politics-a public discussion about the allocation of resources-and it has taken repeated assertion of this fact for the discipline to admit that social science and social scientists are key to implementing and improving the practice of conservation. We have learned much from social science examinations of the nature of power, cross-scale partnerships, institutional structure and environmental governance. We need to better understand the social sciences, we need to appreciate what these disciplines offer and we need social scientists to be more involved in conservation.

Remembering history I Most conservationists were taught that history is evolution. We have suffered a remarkable inability to think about ecological history, let alone human history. The first history we learnt has been termed historical ecology, which has been trying to teach us that humans have been a driving ecological-and even evolutionaryforce for a long time and that notions of pristine nature are not only inaccurate but also have stood in our way as we try to achieve conservation in a human dominated world.

Remembering history II The second type of history we have had to learn is our own history. We have ignored, forgotten or falsely constructed the historical legacy of conservation and then been puzzled that so many of our actions have been rejected by those who not only remember the history but have been victims of it. The imperialist roots of conservation in many parts of the developing world and the forcefully-imposed nature of conservation there have been woven into the fabric of contemporary feelings about conservation practice. This fact was unknown or ignored until impressed upon us by social scientists. Recognizing these historical sensitivities has laid the groundwork for improved practice.

Acknowledging people Conservation is practised by people with a mixture of ethical positions. It is fair to characterize much of conservation, at least in the developed world, as being firmly rooted in a biocentric position. We often see humans as threats to the biological systems we champion. Whereas it is true that the current dismal state of the biosphere is due in large part to accumulated human impact, it is equally true that any success in altering this will require human action. We have been chastised by social scientists for talking about humans only as threats-a persuasive admonition that has contributed to a gradual move towards viewing humans as legitimate elements in nature and an explicit part of the solutions to conservation problems. Social scientists have been largely responsible for bringing to our attention the importance of understanding and more fairly balancing the human costs and benefits of conservation.

The culture of conservation and the culture of conservationists Many conservation practitioners were content to work in the ways we were taught in school: eyes on the immediate path in front of us. We were not reflective practitioners, being too busy addressing the extinction crisis and rushing from one grant, one publication, one project and one challenge to the next to take the time to reflect on our work and our successes and failures. It took criticism from many, including social scientists, for us to recognize that we were both part of the problem and part of the solution and, most importantly, part of a culture that affected the ways we thought and acted and the outcome of our practice. Recognizing our cultural contexts-institutionally, nationally and within our disciplineled to a clearer recognition of our assumptions and therefore of our actions. This was a vital first step in helping to understand what we are doing and understanding how others view our work. From such understanding we hope to bring greater support for conservation.

Valuable social research and interdisciplinary collaboration The social sciences have developed methods to study different aspects of human societies. This has led to rich findings with relevance to conservation and has included such topics as resource tenure, governance, institutions, power, adaptive capacity, human rights and gender. Those interested in more effective conservation can learn from social science research and/or employ social science tools to improve their understanding and practice. We have also learned that poor or cursory social research by untrained biologists may be worse than no research at all and that 
careful, respectful interdisciplinary work has enormous potential and can even develop new fields of enquiry (McClanahan et al., 2009).

\section{Conservation through a glass, darkly: remaining challenges for collaboration}

The second list is a set of generalizations made by some social scientists about the practice of conservation that are incorrect or incomplete. These are not always made explicit but often are. In either case I contend that they warrant more careful examination and that this could lead to both a better understanding of the practice of conservation and a better informed collaboration between conservation practitioners and the social sciences.

Conservation is one thing, with one practice and one set of practitioners Conservation is written about as if it exists as a monolithic practice with identical practitioners. This claim allows a Manichean depiction of the world, with the negative role almost inevitably assigned to conservation: portrayed as undermining the livelihoods of the poorest in pursuit of strictly biocentric aims. However, there are significant differences in conservation organizations, practices and individuals that vary by personal and institutional values, scale, strategies, nationalities, politics, experiences and priorities. Arguments amongst the conservation community in settings such as the World Parks Congress or the Convention on Biodiversity meetings are clear proof of the lack of a single position. The result is a tremendous richness within the practice of conservation that prevents simple generalization. For example, the Wildlife Conservation Society, the organization for which I work, operates in 61 countries with almost 2,000 staff and over 300 projects with a wide range of practices and values. Yet simple generalizations continue to obscure the diversity of conservation, making difficult the collaboration necessary for more effective conservation and potentially impeding the incorporation of more social science into our work. Generalizations obscure the strength of debate within conservation (e.g. between concerns about living diversity in all forms, versus rare or charismatic species, versus the capacity of ecosystems to supply services to humankind). These different concerns lead to different priorities, pursued often by different organizations. These differences matter, and social scientists could help analyse and explain them. For example, community-based conservation is thought by many conservationists to be largely about development and sustainable livelihood promotion and thus not part of the mainstream of conservation. Yet, the definition of what is 'in' and what is 'out' is itself a matter of contention, voice and power. Collaboration built on an understanding of such variation within conservation, its sources, and its consequences would provide a grounded understanding that would help us in our practice and more accurately portray the work of conservation practitioners.

Conservation occurs in epochs The reading of the history of contemporary conservation by some social scientists is frequently shallow. Usually tossed off in a few sentences or a few paragraphs, many authors tell a neat story about the history of conservation that has one strategy replacing the one preceding it with artificial regularity. In some readings, ecoregional-based conservation is said to have been developed in response to the stated failures of community conservation. In others, community-oriented projects have been displaced by payment-based schemes. Although these statements sound compelling, they are neither documented nor appear to have been informed by consultation with those of us who have been involved in these changes. For example, as an early advocate for ecoregional conservation I can say that the efforts with which I was involved had absolutely nothing to do with community conservation. Yet no one has asked. We would benefit from serious attention to detail, informant-based information, variation between places, organizations, individuals: the sort of thing that social scientists are trained to do. Those who examine this history need to recognize that these different approaches rise and fall but never disappear. All serious conservation portfolios contain projects that mix and match different strategies. The modern practice of conservation does change, but its history has not yet been accurately written and the bricolage that currently masquerades as the telling of conservation history is a further disincentive to mutual understanding and collaboration by purporting to define a history that those of us involved know is not correct.

All parks are the same and they are only about the protection of biodiversity The park is often featured as a stereotyped entity. This archetypal park is located in the tropics; people were ejected at the time of its creation and local people are prohibited from using it. It is visited by wealthy tourists from the developed world, run by an autocratic government, enabled by a wealthy international conservation organization, and devoted to the maintenance of large animals in unnatural densities through practices copied from the original inhabitants. That such places exist is indisputable. But all parks are hardly like this. This unfortunate tendency to lump all categories of protected areas under the single appellation of parks and then to define all parks as only about protection of nature is incorrect. As of 2005 there were over 114,000 legally gazetted protected areas registered in the IUCN database (Chape et al., 2008). These fall into six major categories that vary from strict nature reserves to those that protect sustainable use of natural ecosystems. Only $50 \%$ of the total land area under any legal protection is designated for strict protection (categories I-IV; Jenkins \& Joppa, 2009), 
with $41.4 \%$ in areas designed for sustainable use, most with legal human habitation within the protected area (Categories V-VI; Chape et al., 2008). In Italy, for example, the majority of land within protected areas remains under private ownership, with sanctioned agriculture and grazing (Parks.it, 2010). Additionally, recent changes in IUCN's classification of protected areas have created a matrix with multiple governance types ranging from national government to community conserved areas (Dudley, 2008; Dudley et al., 2010). It is clear that there is no more such a single entity as a park as there is such a single entity as a community, as social scientists have demonstrated (Agrawal \& Gibson, 1999). If social scientists differentiate more clearly between different kinds of protected areas it would sharpen their analysis and critique and their impact among conservation professionals.

Conservation is only about parks Conservation is often equated only with parks and the assumption is then made that support for parks means that practitioners think that conservation outside parks is unimportant. Although most conservation practitioners think that protected areas are a vital tool in achieving biodiversity conservation, there is a widespread belief that this is by no means the only tool that must be used. Protected areas are not islands and never were; even focusing on them alone requires working beyond their boundaries. Approaches to conservation that pre-date modern approaches to protected areas are rich and deep and there are extensive literatures that address these approaches. Conservation has never been only about parks and there has been more work done on conservation outside parks than inside them. These conservation tools include land-use planning, resource harvesting, sustainable livelihood promotion, poverty alleviation and human consumption, and there is a rich literature surrounding each of them in both the natural and social science journals. Social science critiques of conservation often underrepresent this work.

Conservation is only a project of the elite inhabitants of the developed world It is true that the contemporary conservation movement has been organized and promoted primarily by elite inhabitants of the developed world and supported by institutions staffed, funded, and/or owned by members of the elite. But the claim that these are the only partisans of conservation is to elide the genuine, widespread and long-standing interest in conservation amongst many groups of people throughout the world. By defining the contemporary conservation movement as only park-based and locating the origin myth in Yellowstone National Park, the argument has become orthodoxy and thus created conditions that deny agency to the many groups such as the First Nations in Canada and their emerging tribal parks movement, the ancient origin of Mongolian protected areas, the increasing recognition of indigenous and community conserved areas, and the sacred connection to protected areas in many of the world's religions. It also denies to citizens of developing nations the right to claim they too are conservationists: not as lackeys of the West, or as a result of colonial determinism, but because they have become convinced of the importance of conservation in this changing world or wish to defend long-standing cultural practices promoting conservation. Women's groups, schools, religious communities, villages, provinces, ethnic groups and nations have demonstrated their commitment to conservation. Conservation is not now, if it ever was, solely an imposed, top-down belief system of elite Western citizens, although this version has had undeniable negative impacts in its execution. Social science could contribute greatly to the understanding of the diverse ways people engage with the ideas and practices of conservation.

Conservation in general and parks in particular are bad for local people Although protected areas have been shown in some cases to be sources of major problems for local residents, this is not always the case and practices are changing fast. There are a significant and growing number of cases ranging from the Chaco of Bolivia to western Canada where the establishment of parks is promoted by local peoples. Park establishment has been used by local peoples to provide tools to help control large-scale mining and agricultural conversion, conserve ways of life, and bring international attention to their culture and problems. Large protected areas declared by local peoples now exist on every continent and many of them are managed for the presence of local cultural and economic activities. Lack of acknowledgement or study of these situations by social scientists writing about conservation denies the complexity that is an inevitable part of conservation as well as the complex ways, positive and negative, that people use protected areas and are, in turn, affected by them.

Marketing of nature is a modern phenomenon Slumbering under the arguments of many who write about trends in conservation is an assumption that in the past nature and natural resources, and the people who lived with them, were not subject to local, regional and international markets. This is an ahistorical rendering of the facts, as there was international trade in products such as obsidian, frankincense, musk, animals for the Roman circuses and ivory for thousands of years, with this trade affecting the resources involved. This trade influenced patterns of human settlement, resettlement, enslavement and occupation long before modern capitalism. Nature was also commodified as hunting, scenery and gardens for thousands of years across most continents. The interplay between nature, commerce and humans is a long, complicated one that denies simple generalization and informs current interests in creating novel markets for nature. There is a need for research into 
the historical understanding of human exploitation of wild species under different economic conditions and over time. The emerging body of work on novel markets and links to globalization is important but needs to be tied to earlier patterns of buying and selling nature.

Conservation in one part of the world is the same as in other parts of the world Much of the most influential literature in this area has been authored by social scientists with experience in eastern Africa but with important contributions from South-East Asia and a few other locations. The experiences from this limited range of places has been generalized to conservation as a whole. Conservation experience from the USA and Europe features much less frequently except when it supports the critique of US national park establishment related to Native Americans. Particularly notable for its low profile in the discussions referenced here is the experience from Europe, where the conservation narrative would seem to differ significantly from much of the rest of the world, and Latin America, where experiences with Native American communities have produced different outcomes. This is not to say that the case made for the East Africa conservation story isn't true but that there is no single truth even from this region, and extrapolation can only be done with exquisite care. Social science could explore how conservation differs in different geographical and social settings with different histories and ecologies.

What is published by conservationists is representative There is a tendency to assign to the entire conservation community the opinions of a few passionate individuals. Other perspectives by these same authors and by many others are passed over in the pursuit of a simple characterization of the whole community. The result has been a synecdoche, a misreading that appeals to other like-minded authors and becomes self-perpetuating and through entrainment of the argument, increasingly inaccurate. There is great diversity of opinion in the community of conservation practitioners and no one speaks for the whole community. Added to this is the fact that most conservation practitioners don't publish in the peer-reviewed literature, if at all. Adjudging what they know and what they think by referring only to the published literature is fraught with dangers. Instead, careful work with this community using the time-tested tools of social science will provide a treasure trove of richness, contradiction, myth and truth - the perfect material for the social scientist.

\section{Discussion}

It will be easy for some to read this piece and dismiss it as yet another attempt by a beleaguered inhabitant of a fortress to strengthen his position. But this would be a misreading of my intentions. I was trained to think that all I needed to know to save the world was biology and it took decades for me to understand how wrong I was. I have come to understand that conservation is about politics and power, about people and societies and history, about morals and values, and about how people view the world and make decisions-all fields of study in the social sciences.

Self-styled as a natural science, conservation is rooted in a set of values and assumptions that privilege the natural science perspective and uses a language to describe itself, its aims, and the rest of the world that reinforces this centrality. However, conservation is not only a natural science: it is a blend of natural and social science, with a large dose of art. People trained in either of these disciplinary traditions need to learn at least to respect, if not to actually use, the methods and theories of the other. From my natural science side we must recognize that just because we are working with people does not mean that we are doing social science.

However, I am not advocating some perfect melding. Growth and improvement often seem to be driven from outside a discipline. It was pointed out by a peer reviewer of this article that fundamental incommensurability between conservation and some other disciplines may be important to acknowledge and possibly to embrace. Conflict and disagreement can be productive and some have argued that the only reason conservation, and some of its practitioners such as myself, have moved in some of the directions indicated here is due to sustained criticism from the social sciences. I am not suggesting some mash-up of disciplines but a more productive engagement-one that brings about improvement-even though that engagement may be criticism. Reyers et al. (2010) advocated broader adoption of transdisciplinary approaches in conservation, arguing that transdisciplinarity '.. . shifts the scientific process from a simple research process that provides a solution, to a social process that resolves problems through the participation and mutual learning of stakeholders (Harorn et al., 2006, in Reyers et al., 2010). This sounds like a powerful way for social scientists to engage with the conservation community.

Geographers, anthropologists and political scientists have been attracted to conservation as a field of study, and more recently as a field of practice, and the social science literature on conservation has increased many fold in the last 20 years. This is an important time for our discipline. Born out of the natural sciences we have come to recognize that conservation can only be effective if it embraces perspectives, values and methods from a wide range of social sciences. This is not happening as well as it should or as rapidly as required. Neither social scientists with life sciences skills nor life scientists with social science skills are being groomed in any number. Unless this changes, we are likely to continue to suffer a split culture of action and critique rather than adaptive practice informed by organized knowledge.

I have taken my hard won appreciation for what the social sciences have to offer conservation back to my 
colleagues in the broader conservation community. There the social sciences are known and appreciated. There are some social scientists who have laboured long and hard within conservation organizations although their expertise has not achieved the necessary change. But ways are changing as conservation careers are being pursued by people trained in the social sciences; even natural scientists are being exposed to the perspectives and approaches of social sciences, as some of the practising social scientists in conservation gain more leverage, as the Social Science Working Group of the Society for Conservation Biology starts to spread its wings, and as more and more of us come to understand that our future success will rely on tools we can learn, borrow or modify from the social sciences. There is still a great deal more to learn.

So this piece is written out of frustration but also out of hope. With all the potential for social science and social scientists to reform and strengthen conservation, aren't there ways to lift the veils obscuring the glass? Lack of progress is due partially to reluctance and neophobia from within the conservation community and a resistance to listen to careful critique. But I would like to suggest that there are many opportunities to improve this relationship that could originate with social scientists. This isn't a new observation. Peter Brosius has pointed out to his fellow anthropologists that their discipline is more interested in identifying what is wrong with conservation than in trying to make things better (Brosius, 2006). Similarly, social science colleagues have observed to me that conservation is now considered a good field in which a young person can gain a reputation by adding to the literature detailing the crimes of conservation. Finding faults-although it can have its place-is easier than suggesting solutions. This serves only to make more difficult the productive engagement of the two areas. But progress has been made with thoughtful openings such as Peterson et al.'s (2008) list of 10 ways that conservation could be employed using a cultural lens perspective derived from anthropology.

The tools that have made the social sciences so perceptive, incisive and helpful to the human condition-the tools that characterize the book by Fairhead \& Leach (1996) - are a means both of improving the practice of conservation and sharpening social science's critique of conservation ideas and practices. With increasing consumption, and demographic, climate and population changes over the next century, conservation will need an even closer engagement with people. To meet this challenge we need careful and informed attention to the details of conservation practice: to variation, power, history, social constraints, and the influence of leaders and how their success depends on the vagaries of social and financial currents; to the role of ego, competition, jealousy and market positioning; to the difference between what is written and what is done; to the power of the institutional eminence gris; to prejudice against one group and favouring of another; to chain migration, happenstance, persuasion and storytelling. These are some of the factors I have seen influence conservation practice. We practitioners are not the monolithic conservation depicted in much of the literature. Those concerned with conservation are humans in pursuit of human ends, with the foibles, powers and weaknesses, and institutional constraints that characterize all human endeavours. Rather than making hasty generalizations about the conservation community based on the writings of the few, and advancing simplistic arguments focused on blame, not improvement, why not study us in our full natural history?

There is a lot of work to be done and a need for systematic analyses of a broad range of case studies, as well as a cessation of the use of case studies as good or bad indications of the worth of the whole. Learning about our practice and the structures that support it would improve our practice. Some, perhaps even most, in the conservation community stand ready. But some will continue to embrace the old ways and in that diversity of approaches will come strength.

In their conclusion Fairhead \& Leach (1996: 3) emphasized the importance of recognizing diverse interpretations of landscapes but warned that: 'Considering all landscape interpretations as in part socially constructed does not, however, negate the fact that certain readings can be demonstrated as false, and that historical evidence might support some more than others'. The themes of diversity of interpretations can mask the veracity of some interpretations over others. Some of what is being said by social scientists about conservation is absolutely true but much of it seems not to be. Although there are many obstacles, there are openings for serious engagement by social scientists with conservationists and the broader conservation community. Researchers are working on institutional ethnographies and placing social scientists in the workplace of conservation organizations. There are probably other similar efforts with which I am not familiar. We need this work. We need to learn of, and from, our mistakes. We must improve our practice. For this, I maintain, we need the help-and informed criticism—of our social scientist colleagues.

\section{Acknowledgements}

I would like to thank my social science friends and colleagues who have taught me so much but are not responsible for what I have learned: Allyn Stearman, Katrina Brandon, Arun Agrawal, Bill Balee, Peter Brosius, Marcus Colchester, Steve Sanderson, Anna Tsing and Paige West. For perceptive and often critical, but always useful comments, I would like to thank Arun Agrawal, Nigel Dudley, Mike Mascia, Dan Miller, John Robinson, Steve Sanderson and Meredith Welch 
Devine. I would also like to thank the constructive engagement of three reviewers.

\section{References}

Agrawal, A. \& Gibson, C.C. (1999) Enchantment and disenchantment: the role of community in natural resource conservation. World Development, 27, 629-649.

Brosius, J.P. (2006) Common ground between anthropology and conservation biology. Conservation Biology, 20, 683-685.

Chape, S., Spalding, M. \& Jenkins, M. (eds) (2008) The World's Protected Areas: Status, Values and Prospects in the 21st Century. University of California Press, Berkeley, USA.

Dudley, N. (ed.) (2008) Guidelines for Applying Protected Area Management Categories. IUCN, Gland, Switzerland.

Dudley, N., Parrish, J.D., Redford, K.H. \& Stolton, S. (2010) The revised IUCN protected area management categories: the debate and ways forward. Oryx, 44, 485-490.

Fairhead, J. \& Leach, M. (1996) Misreading the African Landscape: Society and Ecology in a Forest-Savanna Mosaic. Cambridge University Press, Cambridge, UK.

Harorn, H.G., Bradley, D., Pohl, C., Stephan, R. \& WiESMANN, U. (2006) Implications of transdisciplinarity for sustainability research. Ecological Economics, 6o, 119-128.

JENKINS, C.N. \& Joppa, L. (2009) Expansion of the global terrestrial protected area system. Biological Conservation, 142, 2166-2174.
Mascia, M.B., Brosius, J.P., Dobson, T.A., Forbes, B.C., Horowitz, L., McKean, M.A. \& Turner, N.J. (2003) Conservation and the social sciences. Conservation Biology, 17, 649-650.

McClanahan, T.R., Cinner, J.E., Graham, N.A.J., Daw, T.M., Maina, J., STEAD, S.M. et al. (2009) Identifying reefs of hope and hopeful actions: contextualizing environmental, ecological, and social parameters to respond effectively to climate change. Conservation Biology, 23, 662-671.

PARKS.IT (2010) Http://www.parks.it/indice/Efaq.aree.protette.html\#D10 [accessed 1 September 2010].

Peterson, R.B., Russell, D., West, P. \& Brosius, J.P. (2008) Seeing (and doing) conservation through cultural lenses. Environmental Management, 45, 5-18.

Reyers, B., Roux, D.J., Cowling, R.M., Ginsburg, A.E., Nel, J.L. \& O'Farrell, P. (2010) Conservation planning as a transdisciplinary process. Conservation Biology, 24, 957-965.

\section{Biographical sketch}

Kent H. Redford is Director of the Wildlife Conservation Society Institute at the Wildlife Conservation Society in New York. His work trajectory began in academic settings where he first learned the importance of working with social scientists, to the non-governmental world where he learned the urgency of such collaboration. His interests include the social history of protected areas and their relations with hunting, the practice of conservation, traditional peoples and conservation, and the futures of nature. 